\title{
Development of Quartz Friction Gauge on board Balloon and Sounding Rocket
}

\author{
By Junichi KURIHARA ${ }^{1)}$, Takumi ABE ${ }^{2)}$, Isao MURATA ${ }^{3)}$, Kaoru SATO $^{4)}$ and Yoshihiro TOMIKAWA ${ }^{5)}$ \\ ${ }^{1)}$ Solar-Terrestrial Environment Laboratory, Nagoya University, Nagoya, Japan \\ ${ }^{2)}$ Institute of Space and Astronautical Science, Japan Aerospace Exploration Agency, Sagamihara, Japan \\ ${ }^{3)}$ Graduate School of Environmental Studies, Tohoku University, Sendai, Japan \\ ${ }^{4)}$ Department of Earth and Planetary Science, Graduate School of Science, The University of Tokyo, Tokyo, Japan \\ ${ }^{5)}$ National Institute of Polar Research, Research Organization of Information and Systems, Tokyo, Japan
}

(Received May 2nd, 2008)

\begin{abstract}
The on-board small vacuum gauge developed in this study is the quartz friction gauge, which is based on the principle that the resonance impedance of a quartz oscillator varies with the pressure of ambient gas. The gauge has a wide measuring range $\left(10^{5}-10^{-2} \mathrm{~Pa}\right)$, which corresponds to the atmospheric pressure from the ground to an altitude of about 100 $\mathrm{km}$. The sensor part and data processing part weigh $136 \mathrm{~g}$ and $210 \mathrm{~g}$, respectively. In addition, the gauge can operate at a power consumption level of less than $1 \mathrm{~W}$. The gauge is suited for high altitude balloon experiments where the weight of on-board instruments must be kept to a minimum. The tuning-fork-shaped quartz oscillator in the gauge is widely used for wrist watches and has high resistance to vibration and shock, and thus the gauge is applicable to sounding rocket experiments that require on-board instruments with high resistance to the environment.
\end{abstract}

Key Words: Quartz, Pressure Gauge, Vacuum Gauge, Balloon, Sounding Rocket

\section{Introduction}

Atmospheric pressure measurements in balloon and sounding rocket experiments are technologically and scientifically important. In addition to atmospheric temperature and wind, pressure is one of the important parameters controlling the motion of balloons. In the field of atmospheric chemistry, pressure measurements are of considerable importance in deriving the mixing ratios of important minor constituents such as ozone. In dynamic meteorology, pressure coordinates provide a simpler form of the basic equations and allow for more precise treatments than those in altitude coordinates. Despite the importance, pressure data has been scarcely used for studies of the upper stratosphere $(\sim 50 \mathrm{~km})$, the mesosphere $(50-90 \mathrm{~km})$, and the lower thermosphere $(90-150 \mathrm{~km})$ regions owing to the lack of reliable measurements. Thus a new pressure or vacuum gauge suitable for high-altitude balloon, sounding rocket, and reusable rocket experiments is needed.

The on-board small vacuum gauge developed in this study is the quartz friction gauge, which is based on the principle that the resonance impedance of a quartz oscillator varies with the pressure of ambient gas. The quartz friction gauge was first developed in the 1980's in Japan ${ }^{1)}$ and is now used in laboratories and industries for measurements in low and middle vacuum regions ${ }^{2}$. However, commercial quartz friction gauges are too large and heavy for balloons and sounding rockets. We therefore developed an on-board quartz friction gauge optimized for atmospheric pressure measurements instead of using a commercial quartz friction gauge.

The quartz friction gauge has a wide measuring range from $10^{5}$ to $10^{-2} \mathrm{~Pa}^{3)}$, which corresponds to the atmospheric pressure from the ground to an altitude of about $100 \mathrm{~km}$. The tuning-fork-shaped quartz oscillator in the gauge is very small and light, and is widely used for wrist watches. It also has high resistance to vibration and shock and a long life. The response time of the quartz friction gauge is much quicker than thermal conductivity gauges like the Pirani gauge $^{4)}$. The quartz oscillator itself only requires a power of less than a few $\mu \mathrm{W}$. These features make the quartz friction gauge highly advantageous for use in the on-board gauge. In this paper, we report on the performance tests of the newly developed on-board vacuum gauge and the flight experiments on a high-altitude balloon and a sounding rocket.

\section{Quartz Friction Gauge}

The on-board quartz friction gauge (QFG) consists of a sensor unit and a data processing unit. Fig. 1 shows a flight model of the QFG. The sensor unit and the data processing unit weigh $136 \mathrm{~g}$ and $210 \mathrm{~g}$, respectively.

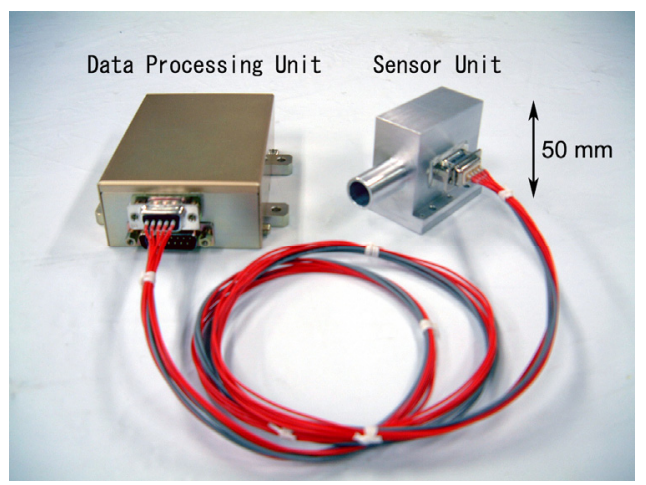

Fig. 1. Picture of the QFG. 


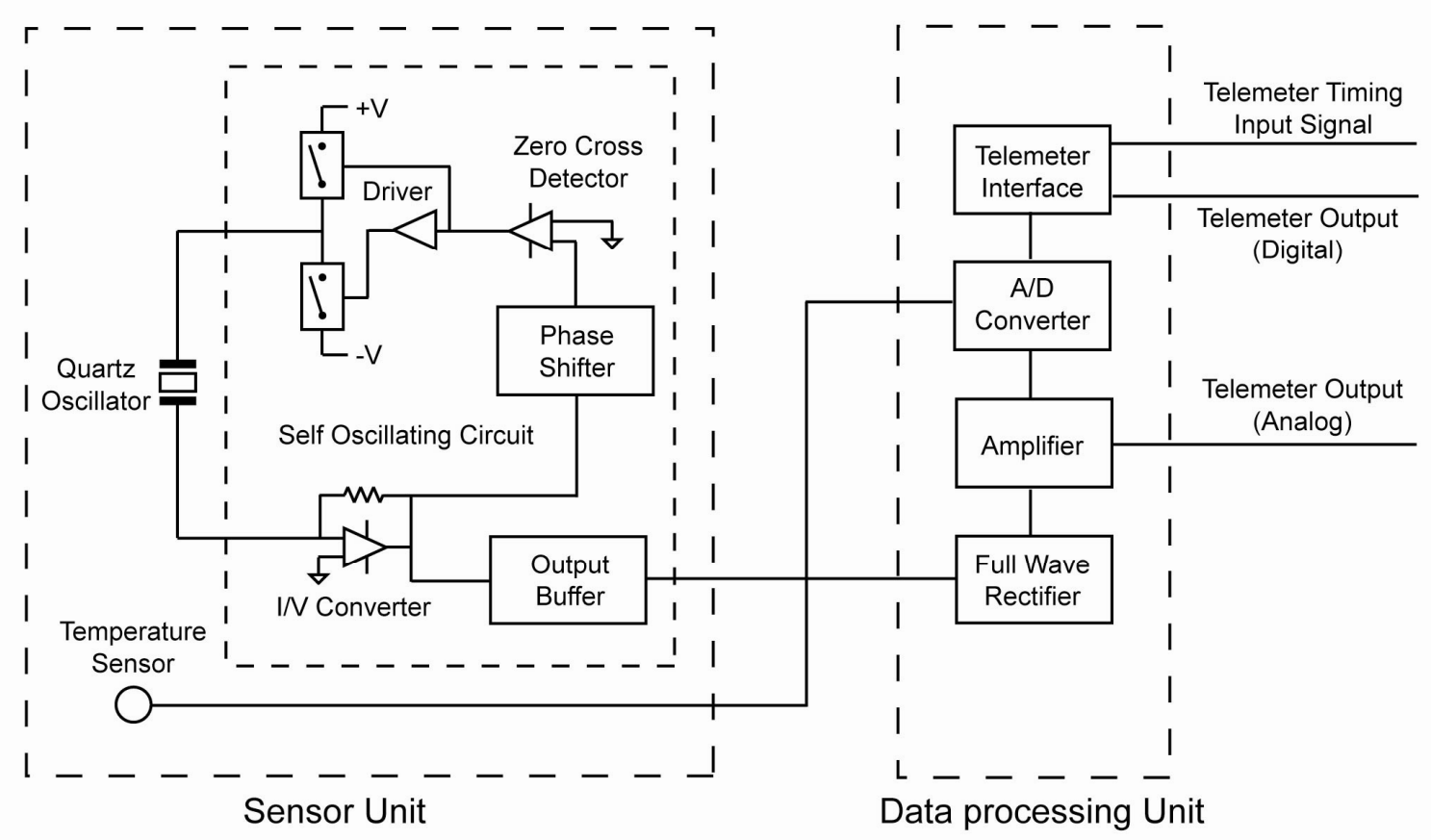

Fig. 2. QFG block diagram.

A block diagram of the QFG is shown in Fig. 2. The sensor unit has a quartz oscillator, an oscillating circuit, and a temperature sensor that was installed in a small metal case with an opening to expose the quartz oscillator to the outside air. This opening has an outer diameter of $15 \mathrm{~mm}$, which is adaptable to the standard gauge port of vacuum systems. The quartz oscillator is a commercially available, tuning-fork-shaped quartz oscillator, which has a resonance frequency of around $32.768 \mathrm{kHz}$ for wrist watches. The current through the quartz oscillator is converted to voltage at the I/V converter in the sensor unit and sent to the data processing unit. In the oscillating circuit, the feedback is provided from the output of the I/V converter to the driver through the phase shifter and the zero cross detector. The temperature sensor is located adjacent to the quartz oscillator and measures temperature of the quartz oscillator. The measured temperature data is used for temperature correction of the pressure measurement.

The data processing unit has an $\mathrm{A} / \mathrm{D}$ converter, an amplifier, a telemeter interface, and a full wave rectifier. The output from the sensor unit is detected at the full wave rectifier and converted to the resonance impedance of the quartz oscillator and amplified to enable transmission of the signal to the telemeter. The QFG can provide both analog and digital outputs to the telemeter in accordance with the plat home.

A total power consumption of less than $1 \mathrm{~W}$ was achieved with this gauge. The detailed power supply voltage, consumption current, and power consumption of each unit are shown in Table 1.
Table 1. Power consumption of the QFG

\begin{tabular}{|r|r|l|r|r|l|}
\hline \multicolumn{1}{|l|}{ Sensor unit } & \multicolumn{1}{|c|}{ Data processing unit } \\
\cline { 1 - 4 } Voltage & Current & Power & \multicolumn{1}{l|}{ Voltage } & Current & Power \\
\hline$+18 \mathrm{~V}$ & $10 \mathrm{~mA}$ & $180 \mathrm{~mW}$ & $+18 \mathrm{~V}$ & $29 \mathrm{~mA}$ & $522 \mathrm{~mW}$ \\
\hline$-18 \mathrm{~V}$ & $10 \mathrm{~mA}$ & $180 \mathrm{~mW}$ & $-18 \mathrm{~V}$ & $4 \mathrm{~mA}$ & $72 \mathrm{~mW}$ \\
\hline$+5 \mathrm{~V}$ & $1 \mathrm{~mA}$ & $5 \mathrm{~mW}$ & & & \\
\hline \multicolumn{3}{|c|}{ Total Power $365 \mathrm{~mW}$} & \multicolumn{4}{c}{ Total Power $594 \mathrm{~mW}$} \\
\hline
\end{tabular}

Table 2. Specification of the QFG.

\begin{tabular}{|c|c|c|}
\hline Pressure measurement range & & $10^{5}-10^{-2} \mathrm{~Pa}$ \\
\hline \multirow{2}{*}{$\begin{array}{r}\text { Pressure measurement } \\
\text { precision }\end{array}$} & $10^{5}-10^{-1} \mathrm{~Pa}:$ & $\pm 10 \%$ \\
\hline & $10^{-1}-10^{-2} \mathrm{~Pa}:$ & $\pm 20 \%$ \\
\hline Operating Temperature range & & $-20-60{ }^{\circ} \mathrm{C}$ \\
\hline Storage Temperature range & & $-20-70^{\circ} \mathrm{C}$ \\
\hline Impact resistance & & $40 \mathrm{G}$ \\
\hline \multirow[t]{3}{*}{ Vibration resistance } & 10-35 Hz: & $3.06 \mathrm{~mm}$ \\
\hline & 35-400 Hz: & $7.5 \mathrm{G}$ \\
\hline & 400-2000 Hz: & $15 \mathrm{G}$ \\
\hline
\end{tabular}

Specifications of the QFG, shown in Table 2, were determined by the requirements for installation on Japanese balloons and sounding rockets operated by ISAS/JAXA. It should be noted that the QFG is able to measure the air pressure even when its temperature is lower than the operating temperature in Table 2. Fig. 3 shows the temperature dependence of the QFG at constant pressure. The quartz oscillator used in the QFG has a temperature dependence following a quadratic function that peaks at about $35^{\circ} \mathrm{C}$. All the pressure data shown in this paper were calibrated with the temperature measured by the temperature sensor. 


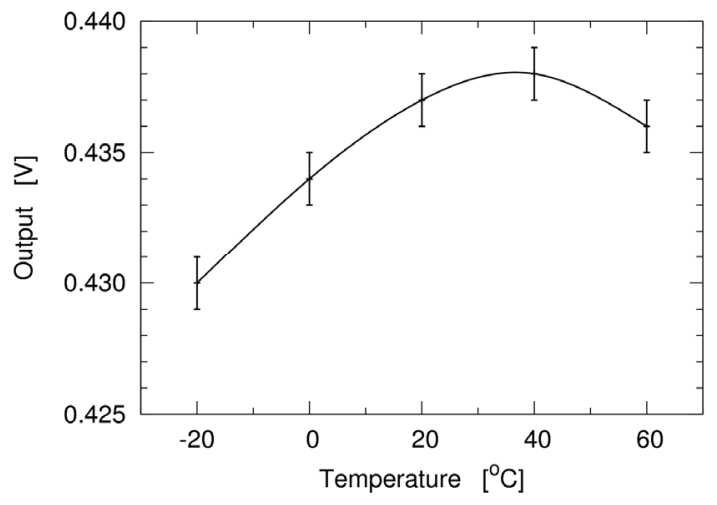

Fig. 3. Temperature dependence of the QFG. output.

The required environmental tests such as the low-temperature, vacuum, shock, and vibration tests were performed at the ISAS facilities. In addition, calibration tests of the QFG were conducted using diaphragm gauges in order to obtain the conversion function for converting resonance impedance of the quartz oscillator into pressure. A set of two or three diaphragm gauges of different measurement ranges was needed for the calibration test because the measurement range of the QFG was 3-4 orders of magnitude wider than that of a single diaphragm gauge. Fig. 4 shows the conversion function obtained by a fifth order polynomial fit to the data. This polynomial function was not used for the post-flight detailed analysis in the present paper because of the poor approximation accuracy in some pressure ranges, but it was useful for the real-time quick-look analysis during the flight. The average error of the polynomial fit is $3.0 \%$. In the post-flight analysis, the conversion was performed by using the linear interpolation of the logarithm of pressure data in Fig. 4.

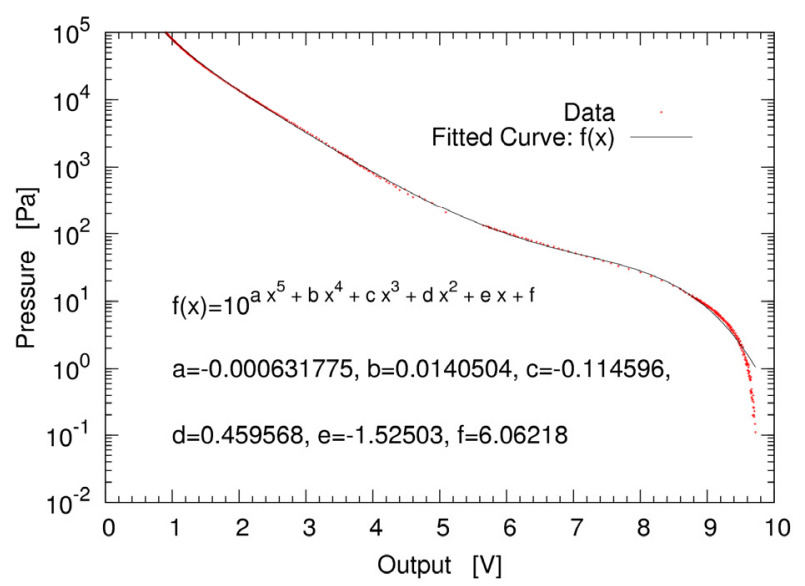

Fig. 4. Conversion function of the QFG output into pressure.

\section{Balloon Experiment}

The flight experiment of the QFG on the balloon BU30-5 was conducted on September 13, 2007. The balloon BU30-5 was launched from the Sanriku Balloon Center in Japan at 6:51 JST (UT +9 hours) and reached an altitude of $49.8 \mathrm{~km}$ at 9:26 JST and landed on the sea about $100 \mathrm{~km}$ away from the launch site at 10:21 JST.

Since the main purpose of this balloon experiment was to observe stratospheric ozone and gravity waves, two instruments, the balloon-borne optical sensor (BOS) and the electrochemical concentration cell ozonesonde (ECC), were carried by the same balloon. The QFG was installed on the BOS payload and utilized the BOS telemeter for data transmission. The BOS and the ECC instruments have diaphragm type pressure gauges and their specifications are shown on Tables 3 and 4, respectively. Simultaneous pressure measurements by the three gauges in this flight experiment were able to provide an important evaluation of their reliability.

Table 3. Specification of the pressure gauge for the BOS.

\begin{tabular}{|r|r|}
\hline Manufacturer & COPAL ELECTRONICS \\
\hline Model name & PA- $830-102 \mathrm{~A}-05$ \\
\hline Measurement range & $981 \mathrm{hPa}-0 \mathrm{~Pa}$ \\
\hline Linearity & $\pm 0.5 \%$ Full Scale \\
\hline Thermal error & $\pm 0.05 \%$ Full Scale $/{ }^{\circ} \mathrm{C}$ \\
\hline
\end{tabular}

Table 4. Specification of the pressure gauge for the ECC.

\begin{tabular}{|c|c|}
\hline Manufacturer & VAISALA \\
\hline Model name & BAROCAP $^{\circledR}$ Silicon sensor \\
\hline Measurement range & $1080 \mathrm{hPa}-3 \mathrm{hPa}$ \\
\hline Resolution & $0.1 \mathrm{hPa}$ \\
\hline \multirow{2}{*}{$\begin{array}{r}\text { Accuracy } \\
\text { (Total uncertainty) }\end{array}$} & 1080-100 hPa: \\
\hline & 100-3 hPa: \\
\hline
\end{tabular}

Fig. 5 shows altitude profiles of atmospheric pressure measured by the QFG and the diaphragm gauges on the BOS and the ECC along with the average pressure derived from the atmospheric model (MSISE-90 $0^{5}$ ). It is important to note that the raw data of the BOS measurement originally had a large offset and thus the BOS data in Fig. 5 was adjusted to the ECC data at the apogee. The ECC measurements agree well with the model up to $40 \mathrm{~km}$. The QFG measurements agree well with the model up to $15 \mathrm{~km}$, but there are large differences $(\sim 30 \%)$ above it.

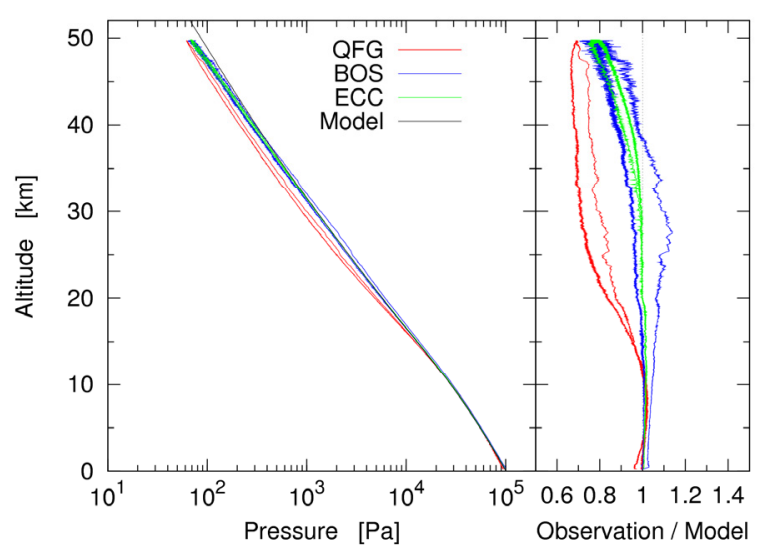

Fig. 5. Altitude profiles of pressure measurements and model. 
These differences can be attributed to the calibration test in the laboratory. As already mentioned, the calibration system uses diaphragm gauges, which measures the total pressure of a gas mixture. On the other hand, the QFG is sensitive to the viscosity of a gas in addition to the pressure, and thus it can be used to measure the composition of the gas mixture ${ }^{2}$. In the calibration test, clean dry air was introduced into the calibration chamber, but the QFG was likely affected by the outgassing from inner surfaces of the chamber and the sensor unit itself.

The QFG and BOS data also have large differences between their ascent and descent measurements $(\sim 10 \%)$ compared to the ECC measurement. These differences between the ascent and descent measurements are attributable to natural pressure variability, but the reason why it was not detected by the ECC is unknown.

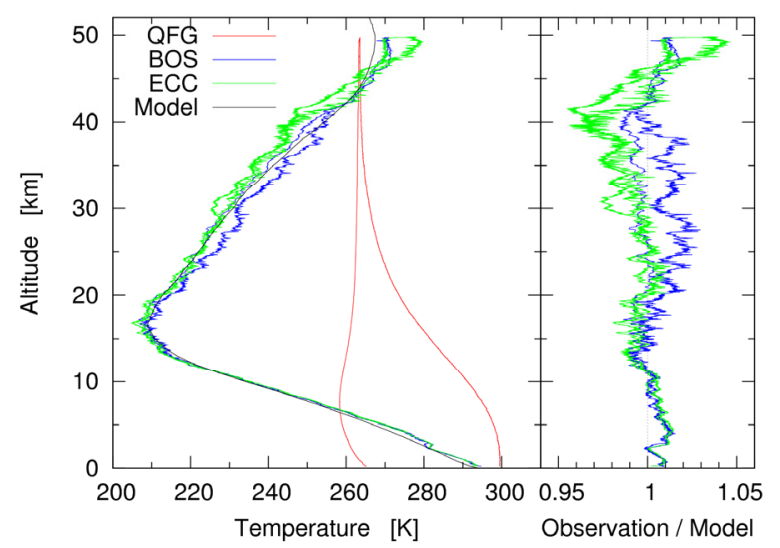

Fig. 6. Altitude profiles of temperature measurements and model.

Fig. 6 shows altitude profiles of the QFG sensor's internal temperature, atmospheric temperature measured by the BOS and the ECC, and average temperature from the model. The QFG sensor temperature slowly follows the atmospheric temperature due to the internal heat sources of the sensor. The pressure data of the QFG shown in Fig. 4 has already been corrected using this QFG sensor temperature data.

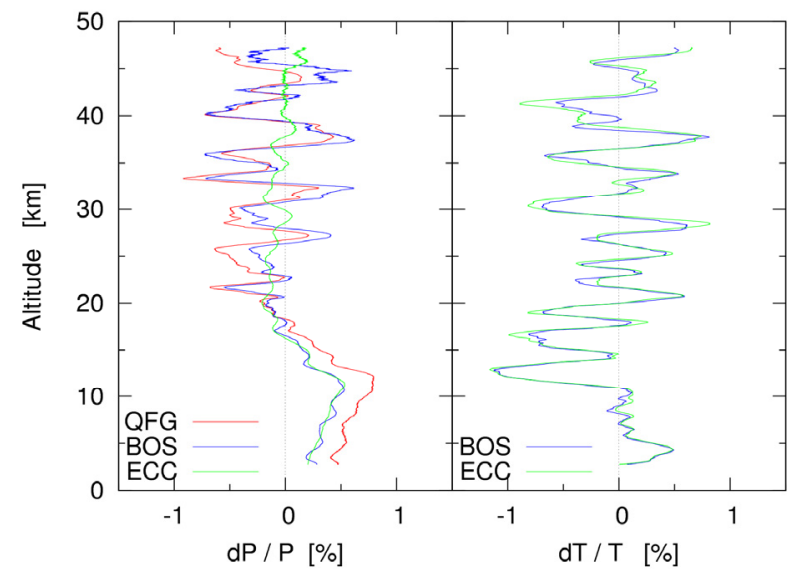

Fig. 7. Fluctuation components of pressure and temperature measurements.
The atmospheric pressures and temperatures shown in Fig. 5 and 6 have fluctuations on their profiles. The fluctuation components in Fig. 7 were extracted from the original profiles using a $1-5 \mathrm{~km}$ band-pass filter. The pressure fluctuations of the three gauges are similar in phase below $20 \mathrm{~km}$ altitude, except for the magnitude of the QFG data. Above $20 \mathrm{~km}$ altitude, the ECC data shows different phases and magnitudes from the QFG and the BOS data. However, the temperature fluctuations of the ECC and the BOS data agree well for all altitudes.

\section{Sounding Rocket Experiment}

The sounding rocket experiment of the QFG was carried out on February 6, 2008. The sounding rocket S-310-38 was launched from the Uchinoura Space Center in Japan at 18:14:40 JST and reached an apogee of $157 \mathrm{~km}$ altitude at 18:17:56 JST. The QFG was installed in the payload section and the nose cone of the payload section was opened $60 \mathrm{sec}$ after the launch at around $72 \mathrm{~km}$ altitude. Since the payload section is not air-sealed completely, the pressure in the payload section decreases with altitude even before the opening of the nose cone.

Fig. 8 shows altitude profiles of pressure measured by the QFG and derived from the atmospheric model. The QFG measurement was not affected by the vibration and shock of the launch except for the noise at around $0.7 \mathrm{sec}$. The measured pressure was consistent with the model up to 55 $\mathrm{km}$ and was almost constant at altitudes above $60 \mathrm{~km}$. This constant pressure of $20 \mathrm{~Pa}$ was maintained by a balance between evacuation and outgassing in the payload section. After the nose cone opening, the pressure measurement stopped due to the saturation of the output. As in the case with the balloon experiment, the saturation can be attributed to the outgassing in the calibration test.

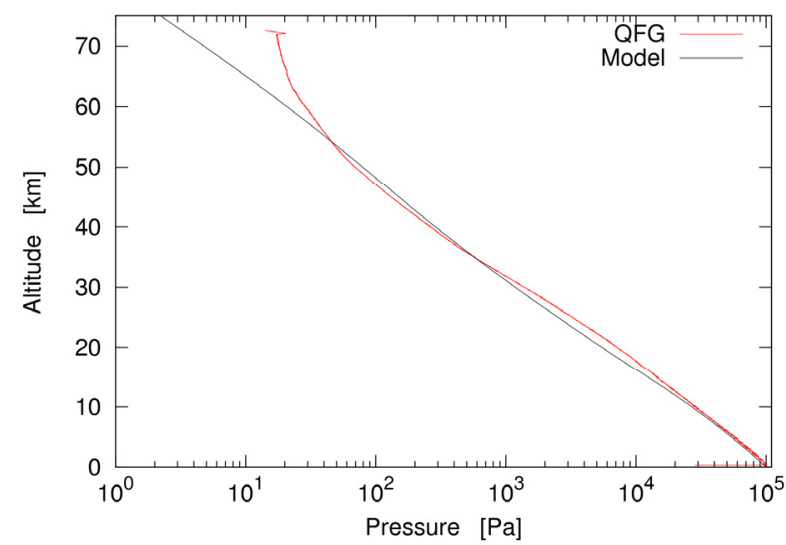

Fig. 8. Altitude profiles of pressure measurement and model.

\section{Conclusion}

The QFG was tested in balloon and sounding rocket experiments. In the balloon experiment, the QFG measurements agreed well with the conventional diaphragm 
gauges and the atmospheric model up to $15 \mathrm{~km}$, but there were large differences $(\sim 30 \%)$ above it. These differences can be attributed to the problem of outgassing in the calibration test. The QFG also measured small pressure fluctuations presumably caused by atmospheric waves. It is suggested that the QFG can be used for studies on atmospheric waves from the troposphere to the mesosphere. The QFG is suited to high-altitude balloon experiments in which the payload weight is severely limited. In the sounding rocket experiment, the QFG measurement was successfully conducted up to $70 \mathrm{~km}$ altitude without failure. Although the measured object in the rocket experiment was not the atmospheric pressure but actually the gas pressure in the payload section, applicability of the QFG in sounding rocket experiments that require on-board instruments with high resistance to the environment has been verified. Accurate pressure measurements by the QFG in a flight experiment require an accurate pre-flight calibration on the ground, with careful attention to the problem of outgassing.

\section{References}

1) Kokubun, K., Hirata, M., Murakami, H., Toda, Y. and Ono, M.: A bending and Stretching Mode Crystal Oscillator as a Friction Vacuum Gauge, Vacuum, 34 (1984), pp.731-735.

2) Suzuki, A., Kurokawa, A., Nonaka, H. and Ichimura, S.: A Possible Hydrogen Sensing Method with Dual Pressure Gauges, Sens. Actuators A, 127 (2006), pp. 37-40.

3) Kobayashi, T., Hojo, H. and Ono, M.: Pressure Measurement from 1 atm to $0.01 \mathrm{~Pa}$ using a Quartz Oscillator, Vacuum, 44 (1993), pp.613-616.

4) Hirata, M., Ono, M., Kokubun, K., Abe, M., Maruno, M., Shimizu, $\mathrm{K}$. and Ogawa, T.: Design and Testing of a Quartz Friction Vacuum Gauge Using a Self-Oscillation Circuit, J. Vac. Sci. Technol. A, 5 (1987), pp. 2393-2396.

5) Hedin, A. E.: Extension of the MSIS Thermosphere Model into the Middle and Lower Atmosphere, J. Geophys. Res., 96 (1991), pp. 1159-1172. 